\title{
Interleukin-17 targeted therapies in axial spondyloarthritis
}

\author{
“...targeting IL-23/IL-17 is a promising and potentially fruitful way for \\ the future of axial spondyloarthritis treatment."
}

Keywords: ankylosing spondylitis • IL-17 •IL-23 • secukinumab • spondyloarthritis

- treatment • ustekinumab

Spondyloarthritis (SpA) is a chronic inflammatory condition, involving mainly the axial skeleton (spine and sacroiliac joints) in young adults [1]. The disease may lead to functional impairment and disability, along with possible structural damage over time that may result in ankylosis. The disease recognizes several phenotypic presentations, including nonradiographic axial spondyloarthritis and radiographic axial spondyloarthritis, in other words, ankylosing spondylitis (AS) [2] .

The pharmacological treatment strategy is based on nonsteroidal anti-inflammatory drugs (NSAIDs), and, eventually, disease modifying drugs for peripheral involvement. In case of inadequate response, the use of anti-TNF agents may be indicated [3]. AntiTNF therapy in spondyloarthritis represents a major breakthrough in the management of the disease, particularly in axial forms [4]. Nevertheless, this treatment is not always effective, and about a third of the patients do not reach adequate response at 3 or 6 months of a first anti-TNF agent [5]. Therefore, there is a need for developing other new targeted therapies in this condition. Progresses in the comprehension of the underlying immunological modifications associated with the disease allow to draw new therapeutic lines of investigation/to explore new therapeutic pathways. The IL-23/Th17 axis is currently considered a main road involved in spondyloarthritis [6]. IL-23 is a cytokine formed by two subunits (p40 subunit common with IL-12 and p19 specific of IL-23), polarizing naive $\mathrm{T}$ cells (expressing IL-23 receptor) toward a Th17 phenotype, characterized by production of IL-17, but also IL-22, leading to inflammation, tissue lesions and production of other cytokines such as TNF. IL-23 is produced by several processes involved in spondyloarthritis: HLA-B27 misfolding inducing an unfolded protein response (UPR) in the endoplasmic reticulum, gut dysbiosis and gut autophagy.

\section{Secukinumab}

Targeting IL-17 in axial spondyloarthritis has come into reality with the evaluation of Secukinumab, a fully human IgG1א monoclonal antibody that selectively binds and neutralizes IL-17A. A first Phase II controlled study exhibited significatively positive results, representing a proof of concept [7], with longterm sustained response and reduction of inflammation on magnetic resonance imaging [8]. Two large Phase III studies (Measure 1 and 2) evaluated secukinumab after either intravenous or subcutaneous loading dose, followed by 75 or $150 \mathrm{mg}$ subcutaneous every 4 weeks over 52 weeks, versus placebo until week 24 . These studies and their results are analyzed in details in a recent issue of immunotherapy [9]. Briefly, secukinumab improved signs and symptoms of the disease, with 60\% of the patients treated with the $150 \mathrm{mg}$ dosage fulfilling an ASAS 20 response at week 16 , versus $28 \%$ in the placebo groups, with sustained response at week 52 . Improvement was also clear in physical function, quality of life and MRI inflammatory scores, with a fair safety profile so far.

These results are interesting, not only as a confirmation of the small proof-of-con-

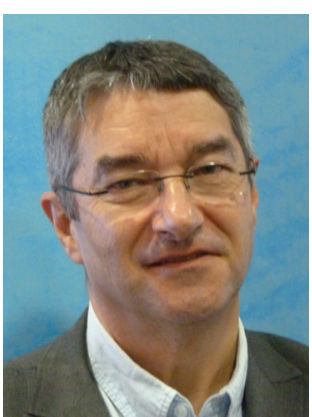

Daniel Wendling

Department of Rheumatology, CHRU de Besançon (University Teaching Hospital), Boulevard Fleming, F-25030 Besançon, France and

Université de Franche-Comté, Boulevard Fleming, F-25030 Besançon, France dwendling@chu-besancon.fr
Future $\because \%$ Medicine part of 
cept study, but also by demonstrating a same range of response (compared with the placebo group) as what is established with the several anti-TNF agents in controlled studies in ankylosing spondylitis [5], even if there are no head-to-head comparative studies available. The second point of interest is the evidence of a similar response with secukinumab $(150 \mathrm{mg})$ both in anti-TNF-naive and anti-TNF-inadequate responder patients (27\% of the patients in Measure 1 and, 38\% in Measure 2). This is an important issue, giving for the first time the answer to an unmet need, demonstrating efficacy after failure of TNF blockade. The third information is the potential role of the loading dose and the dosage of the biodrug on the subsequent response, and perhaps on the development of antidrug antibodies.

Some questions need to be assessed regarding this treatment in axial spondyloarthritis. The efficacy on some rheumatologic feature which may be problematic for the patients, such as enthesitis, dactylitis or peripheral arthritis may be an outcome for evaluation of future studies. In the same way, the impact of this treatment on extra rheumatologic manifestations is important, since they are part of the general presentation of the disease. In non-AS patients, secukinumab showed beneficial effect on uveitis (with high doses) [10], and on skin psoriasis [11]; but the results in Crohn's disease were disappointing [12], even if there are clear connections between gut inflammation and axial spondyloarthritis [13]. The efficacy should also be demonstrated in nonradiographic axial spondyloarthritis, and assessment of the long-term safety and of radiographic progression should be performed.

Some additional studies are ongoing or planned with secukinumab [14] in ankylosing spondylitis. Measure 3 (NCT02008916) is a randomized controlled trial (222 AS patients) evaluating, after intravenous loading dose, 300 or $150 \mathrm{mg}$ subcutaneous every 4 weeks, 16-week efficacy and 3-year safety. Measure 4 (NCT02159053) is an randomized controlled trial (RCT) (324 AS patients) evaluating $150 \mathrm{mg}$ every 4 weeks, with or without subcutaneous loading dose, 16-week efficacy and 2-year safety.

\section{Other agents targeting IL-23/Th17 axis may be used in axial spondyloarthritis}

This may be the case namely for other anti-IL-17A monoclonal antibodies, such as ixekizumab, with investigation programmed in axial spondyloarthritis.

Targeting IL-23 is another interesting option. Ustekinumab is a human immunoglobulin G1к monoclonal antibody that binds to the common $\mathrm{p} 40$ subunit shared by IL-12 and IL-23, developed and approved for skin psoriasis and psoriatic arthritis (PSUMMIT trials). A prospective, open-label, single-arm, proof-ofconcept clinical trial evaluating ustekinumab $90 \mathrm{mg}$ subcutaneously at baseline, week 4 and week 16, was conducted in 20 patients with active AS (modified New York criteria) and BASDAI score of $\geq 4$ despite previous NSAID treatment [15]. ASAS40 response was reached, at week 24 , by $65 \%$ of the patients. ASAS20, ASAS $5 / 6$ and ASAS partial remission were observed in 75, 50 and $30 \%$ of the patients, respectively. $A \geq 50 \%$ improvement of the BASDAI (BASDAI50) occurred in 55\% of the patients. A total of 50 and $20 \%$ of the patients achieved the AS Disease Activity Score (ASDAS) clinically important improvement and major improvement, respectively. At week $24,35 \%$ of the patients had an ASDAS inactive disease (ASDAS <1.3). Significant improvement of other patient-reported outcome parameters and active inflammation as detected by MRI as well as significant reduction of NSAIDs intake were obvious during treatment. Clinical response correlated with reduction of active inflammation on MRI and of serum C-reactive protein level. Overall, ustekinumab was well tolerated.

Studies are planned or ongoing [14], in an attempt to confirm in RCT the efficacy of IL-23 blockade and find the optimal dosage, in patients naive for biodrug, but also in AS patients refractory to one TNF blocker, and also in nonradiographic axial spondyloarthritis. Interestingly, the RCT planned with ustekinumab in AS patients (first two bullets below) have a comparative arm with an anti-TNF agent.

Ustekinumab is evaluated in three Phase III RCT: ustekinumab 45 or $90 \mathrm{mg}$ subcutaneous injection at weeks 0 and 4, followed by every 12 -week dosing:

- Placebo-controlled study evaluating the efficacy and safety of ustekinumab in the treatment of antiTNF- $\alpha$ naive subjects with active radiographic axial spondyloarthritis (NCT02437162), 327AS with elevated CRP and biologic naive;

- Placebo-controlled study evaluating the efficacy and safety of ustekinumab in the treatment of antiTNF- $\alpha$ refractory subjects with active radiographic axial spondyloarthritis (NCT02438787), 483AS elevated CRP, refractory to one anti-TNF;

- Placebo-controlled study evaluating the efficacy and safety of ustekinumab in the treatment of subjects with active nonradiographic axial spondyloarthritis (NCT02407223), 390 non-Rx ax-SpA with positive MRI;

- BI 655066 is an anti-IL-23 p19 monoclonal antibody, with a Phase II study, evaluating in an 
RCT 159 AS patients two doses and placebo (NCT02047110);

- One may use blockade of IL-17 receptor with antiIL-17R antibodies, such as brodalumab, which demonstrated positive results in psoriatic arthritis $[16,17]$, but development was held due to side effects under treatment, a programmed Phase II controlled study, aimed to compare brodalumab $210 \mathrm{mg}$ versus placebo (NCT 02429882), has been cancelled. Targeting IL-23 may also be performed using other anti-p19 IL-23 monoclonal antibodies (guselkumab: CNTO 1959, tidrakizumab : MK-3222), that, in contrast to ustekinumab do not interfere with IL-12; or anti-IL-23 receptor antibodies. Engineering of soluble receptors of IL-17 or IL-23, as well as bispecific monoclonal antibodies (anti-IL-17A and anti-TNF), already tested in inflammatory diseases [18], are other opportunities.

\section{Conclusion}

Once the efficacy and safety is confirmed, the last question to answer is the position of these IL-23/IL-17 targeted therapies in the general strategy of manage-

\section{References}

1 Dougados M, Baeten D. Spondyloarthritis. Lancet 377(9783), 2127-2137 (2011).

2 Wendling D, Claudepierre P, Prati C, Dougados M. Spondyloarthritis: a concept or a disease? Joint Bone Spine doi:10.1016/j.jbspin.2015.06.006 (2015) (Epub ahead of print).

3 Wendling D, Lukas C, Paccou J et al. Recommendations of the French Society for Rheumatology (SFR) on the everyday management of patients with spondyloarthritis. Joint Bone Spine 81(1), 6-14 (2014).

4 Callhoff J, Sieper J, Weiß A, Zink A, Listing J. Efficacy of TNF $\alpha$ blockers in patients with ankylosing spondylitis and non-radiographic axial spondyloarthritis: a meta-analysis. Ann. Rheum. Dis. 74(6), 1241-1248 (2015).

5 Van den Bosch F, Deodhar A. Treatment of spondyloarthritis beyond TNF-alphablockade. Best Pract. Res. Clin. Rheumatol. 28(5), 819-827 (2014).

6 Wendling D, Guillot X, Prati C. The IL-23/Th 17 pathway in spondyloarthritis: the royal road? Joint Bone Spine 82(1), 1-4 (2015).

7 Baeten D, Baraliakos X, Braun J et al. Anti-interleukin$17 \mathrm{~A}$ monoclonal antibody secukinumab in treatment of ankylosing spondylitis: a randomised, double-blind, placebo-controlled trial. Lancet 382(9906), 1705-1713 (2013).

8 Baraliakos X, Borah B, Braun J et al. Long-term effects of secukinumab on MRI findings in relation to clinical efficacy in subjects with active ankylosing spondylitis: ment of axial spondyloarthritis. Should they be used only after failure of anti-TNF agents or as first-line biologic therapy? Only in some subsets of the disease (e.g. with elevated CRP, with radiographic involvement)? This will need further head-to-head and strategy studies.

Obviously, targeting IL-23/IL-17 is a promising and potentially fruitful way for the future of axial spondyloarthritis treatment.

\section{Financial \& competing interests disclosure}

Speaking fees and/or consultancy: Abbvie, MSD, Pfizer, Roche Chugai, BMS, Amgen, Nordic Pharma, Swedish Orphan Biovitrum, UCB. Grants: Abbvie, MSD, Pfizer, Roche Chugai, BMS. National co-ordinator and investigator of the study CAIN 457F2305 (secukinumab in Ankylosing Spondylitis, Novartis). National co-ordinator and investigator of the study ALIGN (sarilumab in Ankylosing Spondylitis, SanofiAventis). The author has no other relevant affiliations or financial involvement with any organization or entity with a financial interest in or financial conflict with the subject matter or materials discussed in the manuscript apart from those disclosed.

No writing assistance was utilized in the production of this manuscript.

an observational study. Ann. Rheum. Dis. doi:10.1136/ annrheumdis-2015-207544 (2015) (Epub ahead of print).

9 Shah R, Perry L, Deodhar A. The role of secukinumab in the treatment of ankylosing spondylitis. Immunotherapy doi:10.2217/IMT.15.93 (2015) (In press).

10 Letko E, Yeh S, Foster CS, Pleyer U, Brigell M, Grosskreutz CL; AIN457A2208 Study Group. Efficacy and safety of intravenous secukinumab in non infectious uveitis requiring steroid-sparing immunosuppressive therapy. Ophthalmology 122(5), 939-948 (2015).

11 Xiong HZ, Gu JY, He ZG et al. Efficacy and safety of secukinumab in the treatment of moderate to severe plaque psoriasis: a meta-analysis of randomized controlled trials. Int. J. Clin. Exp. Med. 8(3), 3156-3172 (2015).

12 Hueber W, Sands BE, Lewitzky S et al. Secukinumab, a human anti-IL-17A monoclonal antibody, for moderate to severe Crohn's disease: unexpected results of a randomised, double-blind placebo-controlled trial. Gut 61(12), 1693-1700 (2012).

13 Wendling D, Vuitton L, Koch S, Prati C. Spondyloarthritis and the gut: a new look. Joint Bone Spine 82(2), 77-79 (2015).

14 ClinicalTrials.gov. www.clinicaltrials.gov

15 Poddubnyy D, Hermann KG, Callhoff J, Listing J, Sieper J. Ustekinumab for the treatment of patients with active ankylosing spondylitis: results of a 28-week, prospective, open-label, proof-of-concept study (TOPAS). Ann. Rheum. Dis. 73(5), 817-823 (2014). 
16 Mease PJ, Genovese MC, Greenwald MW et al. Brodalumab, an anti-IL17RA monoclonal antibody, in psoriatic arthritis. N. Engl. J. Med. 370 (24), 2295-2306 (2014).

17 Bauer E, Lucier J, Furst DE. Brodalumab -an IL-17RA monoclonal antibody for psoriasis and psoriatic arthritis. Expert Opin. Biol. Ther. 15(6), 883-893 (2015).
18 JA, Hueber AJ, Wilson S, et al. Combined inhibition of tumor necrosis factor $\alpha$ and interleukin-17 as a therapeutic opportunity in rheumatoid arthritis: development and characterization of a novel bispecific antibody. Arthritis Rheumatol. 67(1), 51-62 (2015). 